\title{
Simultaneous Measurement of Steering Performance and Perceived Heading on a Curving Path
}

\author{
JONATHAN W. KELLY, ANDREW C. BEALL, JACK M. LOOMIS, ROY S. SMITH, and KRISTEN L. MACUGA \\ University of California, Santa Barbara
}

\begin{abstract}
The ability to judge heading (direction of travel) has been the focus of much research, but a role for perceived heading in steering has not been firmly established. Subjects steered down a road consisting of straight and curved segments and made heading judgments along the way. Heading judgments while traversing curved road segments were biased in the direction of the curve by up to $5^{\circ}$ and position errors on the same curved roads were highly correlated with heading biases. This correlation was revealed by the simultaneous measurement of steering performance and perceived heading.
\end{abstract}

Categories and Subject Descriptors: J.4 [Social and Behavioral Sciences]: Psychology; H5.1 [Information Interfaces and Presentation]: Multimedia Information Systems-Artificial, augmented, and virtual realities

General Terms: Experimentation, Human Factors, Performance

Additional Key Words and Phrases: Heading perception, steering, driving, psychophysics, optic flow

\section{INTRODUCTION}

Gibson [1950, 1958] first suggested that optic flow, or the changing directions of environmental points as one moves through a rigid environment, could be used to successfully control locomotion. When undergoing a pure translation, all points in the optic array emanate from a single point, referred to as the focus of expansion. This focus of expansion corresponds to the instantaneous direction of travel, which has two degrees of freedom when motion through three-dimensional (3D) space is unconstrained (as when flying or swimming). When motion is confined to a horizontal plane (as when walking or driving on the terrestrial plane), the focus of expansion has only one degree of freedom, which is one's heading. ${ }^{1}$ In navigation research and practice, heading is usually specified with respect to a fixed reference direction (e.g., true or magnetic north), but in vision research, it is specified in relation to visible features of the scene. In order to aim toward a target, Gibson noted that one can simply turn so

\footnotetext{
${ }^{1}$ The term "heading" is actually ambiguous. As Loomis and Beall [1998] have observed, there are two common meanings of heading, one referring to facing direction (even of a stationary person or vehicle) and the other referring to travel direction. The aircraft and watercraft navigation literatures refer to the latter as "course," but so as to maintain agreement with the vision research literature, we associate heading with travel direction. Even with this meaning, heading has been used to refer both to current instantaneous direction and direction of future path. In this paper, we define heading as the instantaneous direction of travel.
}

This research was supported by AFOSR grant F49620-02-1-0145.

Authors' address: Jonathan Kelly, Andrew Beall, Jack Loomis, and Kristen Macuga, Department of Psychology, University of California, Santa Barbara, CA 93106-9660; email: \{j_kelly, beall, loomis, macuga\}@psych.ucsb.edu; Roy Smith, Department of Electrical and Computer Engineering, University of California, Santa Barbara, CA 93106-9560; email: roy@ece.ucsb.edu.

Permission to make digital or hard copies of part or all of this work for personal or classroom use is granted without fee provided that copies are not made or distributed for profit or direct commercial advantage and that copies show this notice on the first page or initial screen of a display along with the full citation. Copyrights for components of this work owned by others than ACM must be honored. Abstracting with credit is permitted. To copy otherwise, to republish, to post on servers, to redistribute to lists, or to use any component of this work in other works requires prior specific permission and/or a fee. Permissions may be requested from Publications Dept., ACM, Inc., 1515 Broadway, New York, NY 10036 USA, fax: +1 (212) 869-0481, or permissions@acm.org. (c) 2006 ACM 1544-3558/06/0400-0083 $\$ 5.00$ 


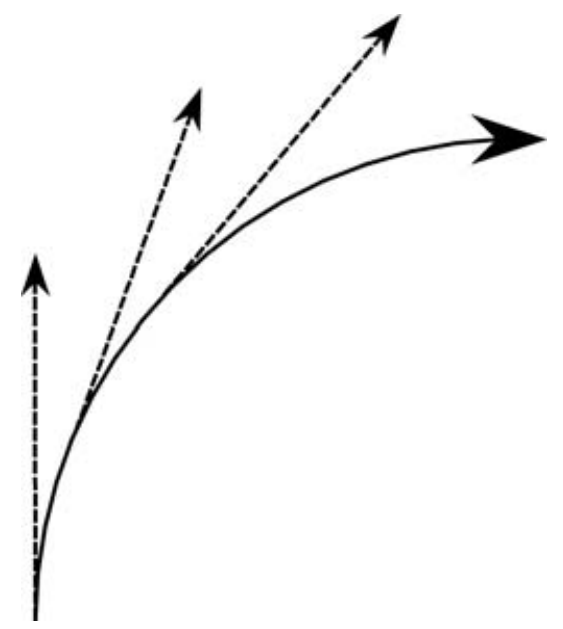

Fig. 1. The solid line represents a hypothetical circular trajectory, and the dashed lines represent an observer's heading at three points along that trajectory. When traveling along this path, heading is constantly changing.

as to place the focus of expansion on the target location, thereby bringing heading into alignment with the direction of the target [Gibson 1950, 1958]. More recent work has made it clear that aiming toward a target, as well as judging heading, does not require that the focus of expansion be present within the visual field [Warren 1976]. Instead, it is now recognized that the global flow pattern, even of just a few points of light, specifies heading.

Since Gibson's proposal, much effort has been devoted to measuring human ability to estimate heading, and most of that effort has focused on the role of optic flow in determining perceived heading (for exceptions, see Beusmans, 1998 and Macuga, Loomis, Beall and Kelly, in press). Careful psychophysical measurements indicate that humans are capable of estimating their heading when traveling along a linear trajectory with an accuracy of 1-2 $2^{\circ}$ [Cutting, Springer, Baren, and Johnson 1992; Royden, Banks, and Crowell 1992; van den Berg 1993; Warren 1998; Warren and Hannon 1988; Warren, Morris, and Kalish 1988]. However, judgments of instantaneous heading along curvilinear trajectories tend to be more error prone, especially at high rotational rates [Royden et al. 1992; Royden, Crowell, and Banks 1994; Stone and Perrone 1997]. ${ }^{2}$ When passively viewing a curvilinear trajectory, heading judgments are biased in the direction of the curve, and these heading biases increase with increasing rotational rates. To explain this bias, Royden [1994] proposed that observers are actually reporting the egocentric direction from themselves to a point on their future path, rather than their actual heading. When traversing a linear trajectory, these two directions, path direction and heading direction, are the same. The discrepancy only arises when taking a curved trajectory, as shown in Figure 1, where instantaneous heading is constantly changing. Contrary to heading judgments, judgments of future path on a curved trajectory are quite accurate [Warren, Mestre, Blackwell, and Morris 1991].

Recently, the role of perceived heading in visually controlled steering has been called into question. In the treatment of this topic, it is important to recognize that visually controlled steering comprises a number of different behaviors that likely require distinct perceptual analyses [Loomis and Beall 1998]. These behaviors include aiming toward a visible target, steering a straight visual path in the presence

\footnotetext{
${ }^{2}$ Although the displays used by Royden et al. [1992, 1994] used simulated observer eye movements while traversing linear trajectories, the instantaneous retinal flow was the same as that of fixed eye gaze along curvilinear trajectories. However, the flow fields in these two cases become distinct when compared over a larger epoch [Lappe, Bremmer, and van den Berg 1999].
} 
of lateral perturbing forces, turning into alignment with a straight path, and steering a curved path. Beall and Loomis [1996] showed that steering a straight path in the presence of perturbing forces can be performed just as well without heading information, as when heading information is available. In addition, Beall [1998; see also Beall and Loomis 1997] provided evidence of a control strategy for turning into alignment with a straight path that did not rely on the sensing of heading information. Both maneuvers rely on the optical orientations (splay) of the edge markers defining the path and their temporal rates of change (splay rate). A steering strategy based on the splay values and splay rates of the edge markers will be referred to as the splay rate strategy. For other work on splay and splay rate in steering, see Chatziastros, Wallis, and Bülthoff [1999], Duchon and Warren [2002], Riemersma [1981], and Mulder, Mulder, and Stassen [2003].

Given two behaviors that do not depend on heading, we can ask about the simple behavior of aiming toward a visible target, for which the research on heading perception prima facie seems most relevant. Instead of using optic flow to judge heading in relation to the direction of the target, an alternate strategy is simply to align one's body (or vehicle) with the egocentric direction of the target [Rushton, Harris, Lloyd, and Wann 1998]. The primary evidence for such a strategy comes from experiments on walking to targets when the sensed egocentric direction differs from the direction to the target specified by optic flow; this dissociation has been achieved using prisms, which optically displace the visual field by a fixed angular amount, or using virtual reality with head-mounted displays. The egocentric direction strategy predicts curved walking trajectories toward the visually offset target, whereas, the optic flow strategy predicts straight trajectories. Many behavioral studies support use of the egocentric direction strategy [Harris and Bonas 2002; Harris and Carré 2001; Rogers and Dalton 1999; Rushton et al. 1998; Turano, Hicks, and Hao 2004; Wood, Harvey, Young, Beedie, and Wilson 2000], but Warren, Kay, Zosh, Duchon, and Sahuc [2001] found that aiming based on optic flow increased in likelihood as the richness of the optic flow information increased, especially when large values of relative motion parallax were produced by numerous vertical poles viewed against a textured ground plane. This increasing dependence on optic flow, however, does not necessarily implicate any role for perceived heading, for optic flow might support alternate control strategies.

We now come to the question of whether heading perception is involved in the most ubiquitous steering task-steering a curved path. The complexity of this behavior depends upon the number of visible targets constraining the path. At one extreme is moving along a curved path to pass through a single point with a specified direction (e.g., skiing through a slalom gate). At the other extreme, is driving a vehicle along a curved road with visible edge markers. The splay rate strategy, which applies to straight paths, does not directly apply to steering a curved path, because the splay values and splay rates depend on which part of the path is being viewed. Similarly, the egocentric direction strategy, which involves aligning one's body or vehicle with the current egocentric direction of a target, seems ill suited to steering a curved path.

A third possible strategy is based on retinal flow (i.e., the flow created from both the changing optic array and eye movements) without the need for computing heading [Kim and Turvey 1999; Wann and Land 2000; Wann and Swapp 2000]. The linearity of the retinal flow lines is highly informative for an observer steering toward a target: when fixating a target, linear retinal flow lines indicate that the target lies on the observer's current path, and curved flow lines indicate over- or understeer, depending on the direction and magnitude of that curvature. In addition, when fixating a target on the future path, the flow lines generated by other points on the path are vertical in the retinal projection. This holds true for both straight and curved trajectories and does not require any estimate of heading. According to this analysis, it is critical that the observer fixates points along the future trajectory and this strategy has, indeed, been demonstrated to improve steering performance [Wilkie and Wann 2003a]. However, by the same token, failure to fixate a point on the future trajectory removes this as a possible steering strategy. 
Still another steering strategy, of course, is to make use of perceived heading. In particular, one might sense heading in order to align one's travel with the current heading of the path. In an investigation of the possible involvement of heading perception, Wann and Wilkie [2006] had observers passively view a curvilinear trajectory, and, occasionally, asked them to fixate either a point on the horizon in the direction of their heading or a point on the ground plane that lay on their future path. Errors in judging heading were three times larger than errors in judging path. They concluded that heading information is not available and, therefore, cannot be used to guide locomotion. Path perception, on the other hand, was quite accurate, which supports the general idea presented above, that path fixation is critical in controlling steering.

Although evidence is mounting that perceived heading may not be important in the visual control of steering, it has yet to be shown that this is the case in an active control task. Thus, in the following experiment, observers steered a vehicle along a curving path and made heading judgments along the way. Given that perceived heading when traversing a curve has been shown to be biased, this experiment explores the correlation between these heading biases and steering performance. If heading is important in steering, then any bias in heading judgments ought to have deleterious effects on steering performance. In addition, sinusoidal crosswinds perturbed the vehicle as observers steered down the path and larger crosswinds should have a greater impact on steering performance. These crosswinds were added to further elucidate the relationship between heading and steering: while manipulation of road curvature was intended to primarily affect perceived heading, manipulation of crosswind magnitude was intended to affect steering performance. Furthermore, the crosswinds required observers to actively control the vehicle at all times.

\section{METHODS}

\subsection{Participants}

Eight graduate students at the University of California, Santa Barbara were paid for their participation. All participants were verified to have a minimum of $20 / 20$ vision and at least $80 \%$ stereopsis as measured by a Keystone orthoscope.

\subsection{Design}

In a $3 \times 3$, fully-factorial design, there were three levels of road curvature and three levels of a perturbing crosswind. Subjects drove nine roads total, each for approximately 8 minutes. While road curvature varied randomly within all nine of the roads, wind magnitude was blocked, such that subjects drove three roads with one wind magnitude, then three roads with another wind magnitude, etc. The order of wind magnitude was randomized across subjects. Subjects made 30 heading judgments of varying difficulty for each of the nine combinations of road curvature and crosswind magnitude.

Prior to starting the experiment, subjects drove a practice road for 4 minutes. Subjects drove the practice road in the presence of an intermediate crosswind and made relatively easy heading judgments to make sure they understood the task. No feedback was provided at any point during the experiment.

\subsection{Stimuli}

All roads were created by combining three types of road segments: straight segments, low-curved segments, and high-curved segments (Figure 2). Each road segment was $19.8 \mathrm{~m}$ (11 eye-heights) long and $3.96 \mathrm{~m}$ (2.2 eye-heights) wide. The low- and high-curved segments had curvature radii of $37.8 \mathrm{~m}$ (21 eye-heights) and $18.9 \mathrm{~m}$ (10.5 eye-heights), respectively. This resulted in low-curved segments that changed direction by $30^{\circ}$ and high-curved segments that changed direction by $60^{\circ}$ over the length of the segment. Thus, when traveling at $3.96 \mathrm{~m} \mathrm{~s}^{-1}\left(2.2\right.$ eye-heights $\left.\mathrm{s}^{-1}\right)$, heading changed at a rate of $6^{\circ} \mathrm{s}^{-1}$ 


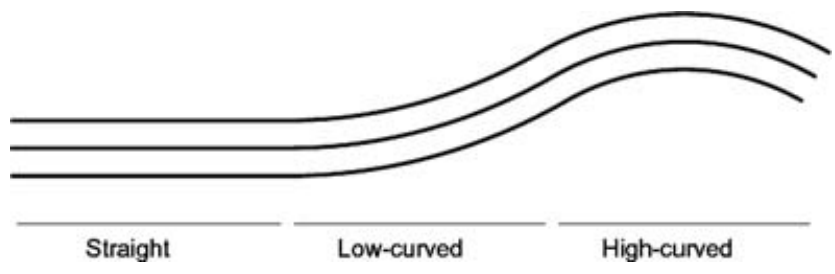

Fig. 2. Road segments used to build experimental roads.
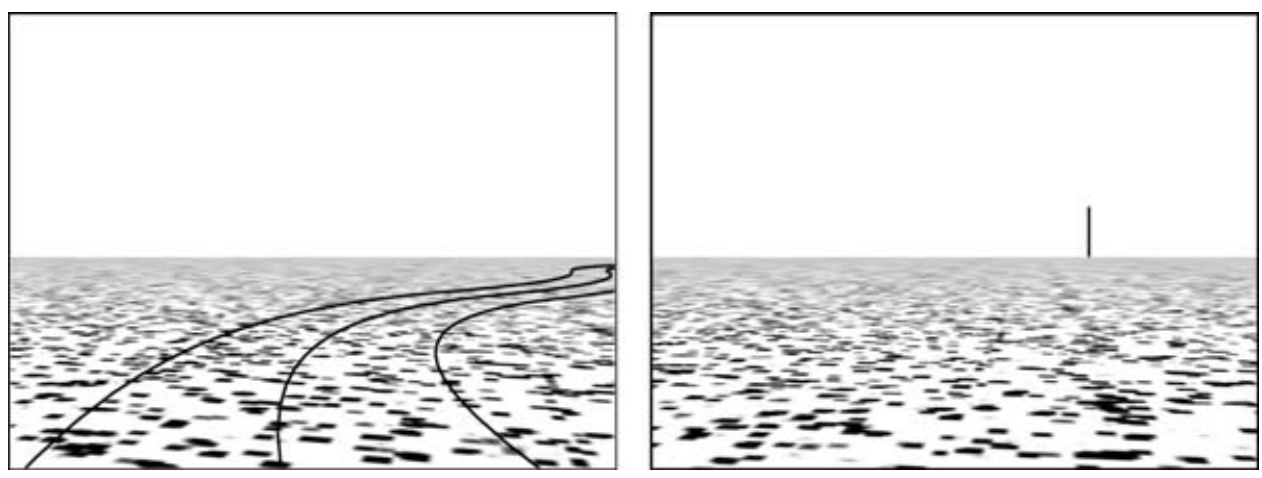

Fig. 3. Single frame of the experimental display during the steering task (a) and during the heading task (b). In the actual display, the ground texture was black with white texture elements, the path lines were bright green, and the heading probe was red.

and $12^{\circ} \mathrm{s}^{-1}$ when steering the low- and high-curved segments, respectively. Eye-height was simulated at $1.8 \mathrm{~m}$.

Roads were constructed by adding the three road segment types (straight, low-curved, and highcurved) in blocks of three segments arranged in a pseudorandom order. The only constraint was that a given segment was never connected to a segment of the same type (i.e., the third segment of one block was never the same as the first segment of the next block). The direction of the curves (leftward or rightward) was random. A total of nine roads were created in this manner, each road consisting of 150 segments. The ground plane was a random dot texture, with white dots scattered over a black ground plane. The road was drawn with three bright green lines: a centerline and two lane markers, which were clearly visible on the black ground plane. Figure 3a displays a single frame of the road as seen from the observer's perspective (note that the colors and contrasts have been altered for publication purposes). The road was visible only out to a distance of $72 \mathrm{~m}$ (40 eye-heights) from the observer to prevent spatial aliasing of the distant lane markers with the graphics raster.

Sinusoidal "crosswinds" perturbed the vehicle heading with a period of $12 \mathrm{~s}$. This gave the impression of a lateral force causing the vehicle to turn slightly (rather than slip sideways). At peak amplitude (and in the absence of any compensatory steering input by the subject), the steering perturbations resulted in heading changes of $12^{\circ}, 24^{\circ}$, and $36^{\circ} \mathrm{s}^{-1}$. These perturbations are, henceforth, referred to as low, medium, and high crosswinds.

\subsection{Materials}

To eliminate any orientation cues provided by the outline of a stationary video display, stimuli were viewed through a Virtual Research (V8) head-mounted display (HMD) with $640 \times 480$ resolution LCD panels refreshed at $60 \mathrm{~Hz}$. The visual graphics were also updated at $60 \mathrm{~Hz}$ for both eyes. The field of view 
was $50^{\circ}$ horizontal by $38^{\circ}$ vertical. Projectively correct stereoscopic images were rendered by a dual 800 $\mathrm{MHz}$ processor computer with a nVidia Quadro4 550 XGL graphics card. Observer head orientation was measured with a three-axis orientation sensor (Intersense IS300) and recorded at $60 \mathrm{~Hz}$. Head orientation data was used to update the perspective images sent to the HMD. Subjects were encouraged to keep their heads relatively still, but slight head movements that inevitably occur rendered the outline of the visual display less reliable for judging heading within the virtual environment. If a strong frame of reference fixed to the vehicle (like a windshield) were visible to the subject, they could use the reference frame to determine their heading directly. In this experiment, no such reference frame was present.

Steering was controlled with a Logitech Momo Racing steering wheel with a mild centering spring. When probed for heading judgments, subjects responded by pressing either of two buttons on the steering wheel.

\subsection{Procedure}

Subjects were seated and steered along roads consisting of straight, low-curved, and high-curved road segments, with a forward velocity of $3.96 \mathrm{~m} \mathrm{~s}^{-1}\left(2.2\right.$ eye-heights $\left.\mathrm{s}^{-1}\right)$. The vehicle had a maximum turn rate of $60^{\circ} \mathrm{s}^{-1}$ and had perfect friction. Deflection of the steering wheel resulted in a change of vehicle heading and a resultant change of vehicle position, much like a standard car. Unlike a typical car, the simulated vehicle rotated around a point centered at the location of the subject, neglecting the more complicated vehicle dynamics, such as wheelbase. For each heading judgment, one of the three road segment curvatures was randomly selected by the computer to be probed next. Once the subject arrived at a road segment of the selected curvature, they continued to steer along that segment for 2 seconds, and were then asked to make a judgment of their heading. Subjects were given as much time as they needed to make this judgment. During a heading judgment, the vehicle was frozen, the green road lines were removed from view (the textured ground plane remained visible), and a heading probe appeared at the horizon (Figure $3 \mathrm{~b}$ ). The heading probe was a red bar, which subtended (in units of visual angle) $0.25^{\circ}$ horizontally and $4^{\circ}$ vertically. The heading probe could appear $1^{\circ}, 2^{\circ}, 4^{\circ}, 8^{\circ}$, or $12^{\circ}$ to the left or right of the observer's actual heading. This angular deviation is referred to as the heading angle. The observer was instructed to decide whether their instantaneous heading, just prior to when the vehicle was stopped, was to the left or right of the heading probe. Observers were explained that their instantaneous heading was the direction that their vehicle would go if the steering wheel was immediately returned to center and the vehicle continued to move straight forward. Responses were entered by the observer by pressing a button on the steering wheel. After completing the heading judgment, the vehicle accelerated at a rate of $1.98 \mathrm{~m} \mathrm{~s}^{-2}\left(1.1\right.$ eye-heights $\left.\mathrm{s}^{-2}\right)$ until it reached maximum forward velocity $\left(3.96 \mathrm{~m} \mathrm{~s}^{-1}\right)$.

For each of the nine roads (three roads for each of the three crosswind magnitudes), subjects made 30 heading judgments, consisting of 10 heading judgments (one for each possible heading angle to the left and right of their actual heading) on each of the three path curvatures. This took approximately 8 minutes to complete. Subjects, therefore, made a total of 270 heading judgments.

While driving the same roads, subjects attempted to stay directly over the centerline of the road. Position error relative to the centerline was recorded throughout the experiment at $60 \mathrm{~Hz}$.

\section{RESULTS}

\subsection{Heading Judgments}

When steering curved path segments, observers' heading judgments were biased in the direction of the curve. Given this bias, data from leftward and rightward curves must be kept separate for analyses. Unfortunately, the experimental design was limited in that curve direction was not independently 

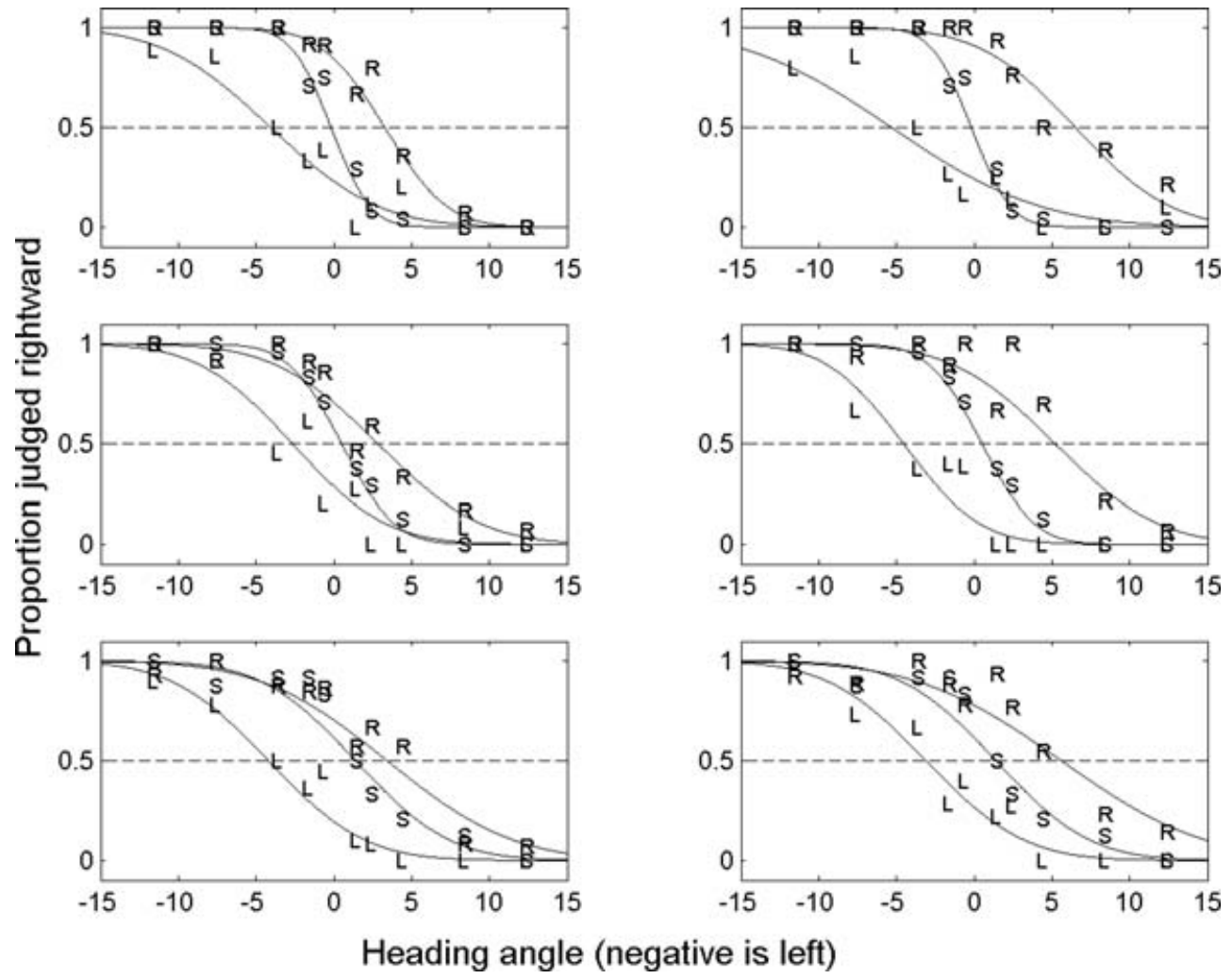

Fig. 4. Mean heading judgments while steering low-curved (left column) and high-curved (right column) road segments, in the presence of low (top row), medium (middle row), and high (bottom row) crosswinds. Proportion of rightward heading judgments (i.e., when subjects believed their heading was to the right of the heading probe) is plotted as a function of the location of the heading probe (in degrees). Data for straight, leftward-, and rightward-curved road segments are indicated with "S," "L," and "R," respectively.

varied, resulting in incomplete heading judgment data for some subjects. Average heading data and probit functions fit to those average data are presented in Figure 4. Performance is quite good for large heading angles-both positive and negative. This is evidenced by the probit functions in Figure 4, which are close to one for negative heading angles and close to zero for positive heading angles. As the heading angles approach zero, performance degrades considerably. There are three general findings to be drawn from Figure 4. First, heading judgments are biased in the direction of the curve (low-curve heading data are presented in the left column and high-curve heading data are presented in the right column). This bias is revealed by the leftward and rightward shifts of the probit functions in Figure 4 for heading judgments made on leftward and rightward curves, respectively. Second, heading judgments are less sensitive when steering curved segments, revealed by the shallower slopes of the probit functions for heading judgments made on curved segments compared to straight segments. Third, heading judgments are less sensitive when steering with larger wind magnitudes, shown by the shallower slopes of the probit functions for increasing wind magnitudes (low, medium, and high wind data are presented in the top, middle, and bottom row, respectively).

Although the heading biases in Figure 4 appear to increase with increasing curve magnitude, these biases are roughly constant with increasing wind magnitude. Since heading bias is constant with 


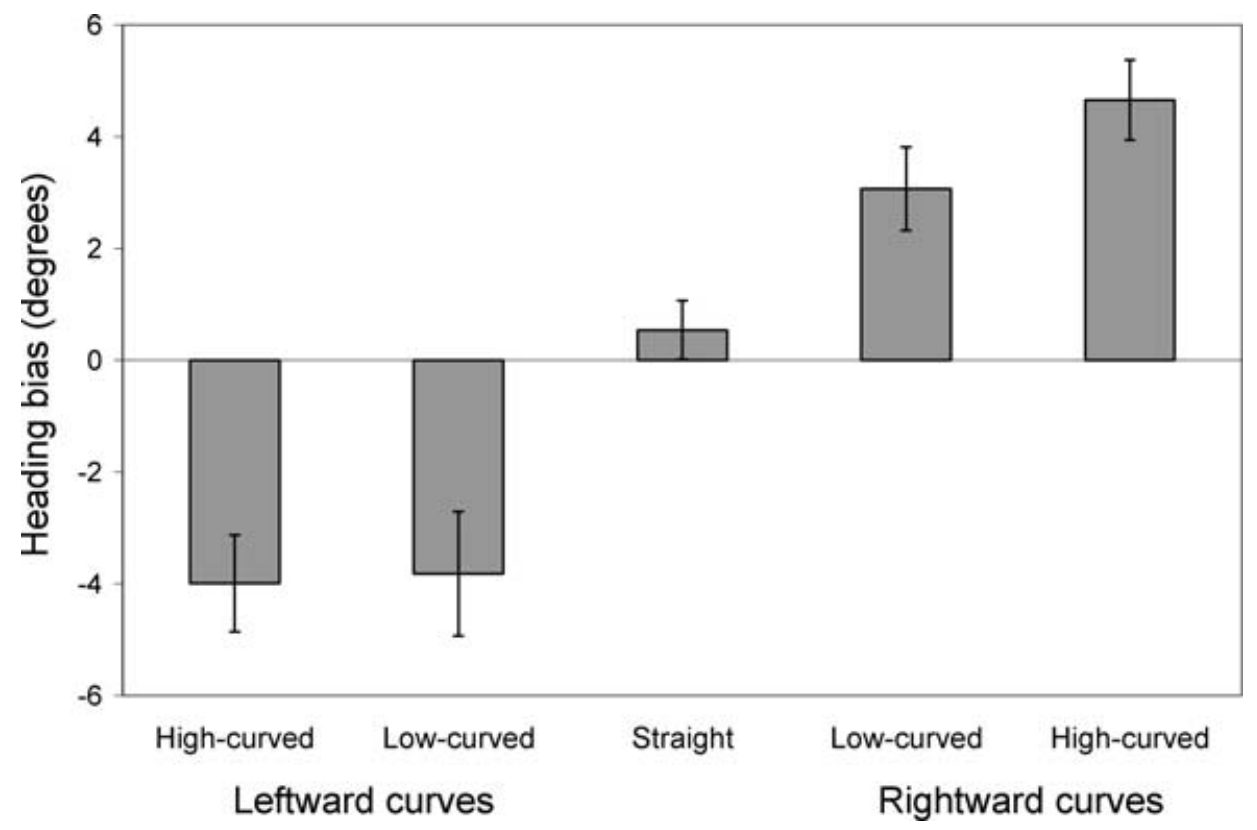

Fig. 5. Heading biases when steering the different path segments. A negative heading bias is a leftward bias and a positive heading bias is a rightward bias from the driver's egocentric frame of reference. Error bars represent \pm 1 standard error.

increasing wind magnitude, each observer's data was averaged across wind magnitude. This was done in order to obtain full data sets for individual observers, which is necessary for proper statistical analysis of the heading bias. Probit functions were then fit to each observer's average data and used to calculate the point of subjective equality (PSE) for heading judgments. The PSE is the heading angle, which subjects judged to be left of their actual heading $50 \%$ of the time and, if significantly different from zero, is considered a bias in judging heading. Figure 5 plots this bias for the different road segment curvatures, keeping leftward and rightward curves separate. Clearly, heading judgments are biased in the direction of the curve being steered. A one-way repeated measures ANOVA reveals a main effect of curvature, $F(4,28)=30.35, p<0.01$.

In addition to this bias, heading judgments are less sensitive when steering curved paths. Unfortunately, since sensitivity also appears to decrease with increasing wind magnitude, it is not appropriate to average observer data across wind magnitude in the analysis of heading sensitivity. Therefore, statistical analyses cannot be performed on this data and meaningful conclusions cannot be drawn regarding heading sensitivity and path curvature. The same problem prevents analysis of the trend for decreasing heading sensitivity with increasing wind magnitude.

\subsection{Steering Performance}

Position error of the vehicle was measured at the same moment that subjects made the heading judgment ( $2 \mathrm{~s}$ into the curve). With regard to steering performance, root-mean-squared (RMS) position error, expressed as a percentage of road width, increased with increasing road curvature as well as increasing wind magnitude (see Table I). RMS error indicates the precision of the observer's position on the road. Since the RMS errors for leftward and rightward curves are quite similar, the RMS data were averaged across curve direction in order to conduct the appropriate statistical tests. A two-way repeated-measures ANOVA was conducted to evaluate the effects of road curvature (straight, low, and high) and wind 
Table I. RMS Position Error Expressed as Percentage of Road Width, for all Combinations of Road Curvature, Curve Direction, and Wind Magnitude

\begin{tabular}{|c|c|c|c|c|c|}
\hline & \multicolumn{2}{|c|}{ Leftward Curves } & \multirow[b]{2}{*}{ Straight } & \multicolumn{2}{|c|}{ Rightward Curves } \\
\hline & High-curved & Low-curved & & Low-curved & High-curved \\
\hline Low wind & $7.9 \%$ & $5.6 \%$ & $4.1 \%$ & $4.6 \%$ & $7.5 \%$ \\
\hline Medium wind & $8.8 \%$ & $6.3 \%$ & $4.9 \%$ & $5.9 \%$ & $8.6 \%$ \\
\hline High wind & $10.1 \%$ & $8.5 \%$ & $6.0 \%$ & $6.3 \%$ & $8.2 \%$ \\
\hline
\end{tabular}

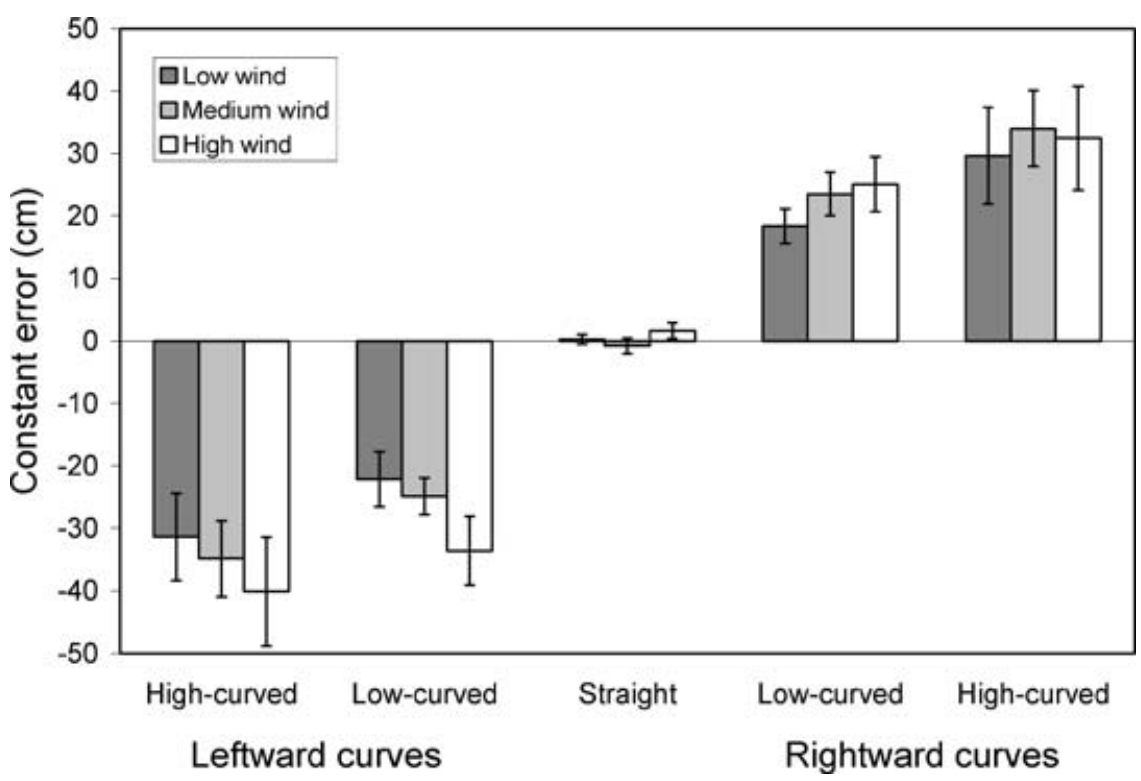

Fig. 6. Constant (mean) position error, broken down by both crosswind magnitude and path curvature. A negative position error is an error toward the left side of the road and a positive position error is an error toward the ride side of the road. Error bars represent \pm 1 standard error.

magnitude (low, medium, and high) on RMS position error. The main effect of road curvature was significant, $F(2,14)=9.84, p<0.01$, as was the main effect of wind magnitude, $F(2,14)=5.35, p<0.05$. There was no significant interaction between road curvature and wind magnitude. Mean RMS position errors were correlated with unsigned heading biases, ${ }^{3} r=0.57, p<0.05$.

Although RMS error is the traditional measure of performance in the driving literature, it cannot reveal a mean position error. Since RMS error is unsigned and calculated relative to the center of the road, it is unclear whether the errors observed in Table I are due to mean position error or to variability in the position data. Therefore, in order to fully describe steering performance, the position data was also analyzed as the observer's mean position with respect to the center of the road. This was done to determine whether observer position was biased toward the inside or the outside of the curves. Figure 6 presents mean position for leftward and rightward turns and shows that observers steered toward the inside of the curves. This is seen in the negative mean position for leftward curves and the positive mean position for rightward curves. A two-way repeated-measures ANOVA revealed a main effect of road curvature, $F(4,28)=30.80, p<0.01$ and no effect of wind magnitude,

${ }^{3}$ Unsigned heading bias was used to calculate this correlation because RMS position error is, by definition, also unsigned. 
$F(2,14)=0.74$, ns. The interaction between road curvature and wind magnitude was not significant, $F(8,56)=2.42$, ns. Mean constant errors were highly correlated with heading biases, $r=0.95$, $p<0.01$.

\section{DISCUSSION}

While others have shown heading biases during passive observation of curvilinear trajectories [Stone and Perrone 1997], this experiment represents the first attempt to simultaneously measure steering performance and perceived heading. The primary finding is that heading judgments were biased in the direction of the curve being steered and that these biases were closely correlated with in-lane position errors. Most popular control models of steering place significant emphasis on heading [Donges 1978; Godthelp 1986; McRuer, Allen, Weir, and Klein 1977], but some have proposed that locomotion can be controlled without the use of heading [Land 1998; Wann and Wilkie 2004b; Wilkie and Wann 2003b]. Generally speaking, these alternative strategies are based on the bearing of a target location (i.e., the angular direction of the target relative to one's facing direction), or the rate of change of that bearing over time. Still, advocates of heading-based control strategies have argued that even these models require heading implicitly (see Fajen and Warren, 2000, and Wann and Land, 2000, 2001, for presentation of both sides of the argument).

While the heading errors seen in this experiment were highly correlated with position errors, it is still possible that this relationship is not causal. Rather, both types of errors could be due to some feature of curved roads. One possibility is that subjects erroneously used a steering strategy based on the vehicle's position in the lane. If subjects attempted to steer the vehicle by aiming toward the nearest visible portion of the lane marker, they would turn too sharply and exhibit position errors similar to those seen in Figure 6. In a real car, this would be like keeping the hood ornament of the car aligned with the lane marker visible above the hood. Although there was no hood ornament (or any other visible vehicle reference frame) in this experiment, subjects could have used the HMD as an equivalent frame of reference. However, subject head movements during the experiment rendered the HMD reference frame less reliable.

Alternatively, the position errors could potentially be because of the simulated vehicle model used in the experiment. The dynamics of the vehicle model were different from those of a typical car in that the simulation did not take friction or wheelbase into account. Rather, when the subject was moving forward and turned the steering wheel, the vehicle responded by rotating about a point centered on the subject (a commonly used vehicle model in vision research; Beall and Loomis, 1996, Wilkie and Wann, 2003a). It is possible that subjects steered the vehicle as though they were driving a real car with a complete set of dynamics, potentially leading to steering errors. However, subjects received 4 minutes of practice to familiarize themselves with the vehicle control, and none reported any confusion or surprise in response to the simulated vehicle dynamics. In addition, most drivers find it fairly simple to drive a new car with quite distinct dynamics from the car they are most familiar with, suggesting that humans are quite adept at internalizing the dynamics of a new vehicle in a short amount of time.

Given the possibility that steering errors and errors in perceived heading could arise independently, further experiments where heading perception is experimentally degraded or biased could prove useful in determining the exact relationship between perceived heading and steering.

\section{ACKNOWLEDGMENTS}

This data was presented at the Vision Sciences Society meeting, May 2004. We thank John Wann and two anonymous reviewers for helpful comments. 


\section{REFERENCES}

BEALl, A. C. 1998. Visual control of the base-to-final turn in fixed wing aircraft. PhD thesis, University of California, Santa Barbara, CA.

BeALl, A. C. AND Loomis, J. M. 1996. Visual control of steering without course information. Perception 25, 481-494.

Beall, A. C. and Loomis, J. M. 1997. Optic flow and visual analysis of the base-to-final turn. Int. J. Aviation Psychol. 7, $201-223$.

Beusmans, J. M. H. 1998. Perceived object shape affects the perceived direction of self-movement. Perception 27, $1079-1085$.

Chatziastros, A., Wallis, G. M., and Bülthoff, H. H. 1999. The use of splay angle and optical flow in steering a central path. Max Planck Institute for Biological Cybernetics, Technical Report 72.

Cutting, J. E., Springer, K., Baren, P. A., AND Johnson, S. H. 1992 . Wayfinding on foot from information in retinal, not optical flow. J. Exp. Psychol. Gen. 102, 41-72.

Donges, E. 1978. Two-level model of driver steering behavior. Hum. Factors 20, 691-707.

Duchon, A. P. AND WarRen, W. H. 2002. A visual equalization strategy for locomotor control: Of honeybees, humans, and robots. Psychol. Sci. 13, 272-278.

Dyre, B. P. And Andersen, G. J. 1997. Image velocity magnitudes and perception of heading. J. Exp. Psychol. Hum. Percept. Perform. 23, 546-565.

Fajen, B. R. AND WarRen, W. H. 2000. Go with the flow. Trends Cog. Sci. 4, 369-370.

Gibson, J. J. 1950. Perception of the Visual World. Houghton Mifflin, Boston, MA.

Gibson, J. J. 1958. Visually controlled locomotion and visual orientation in animals. Br. J. Psychol. 49, $182-194$.

Godthelp, H. 1986. Vehicle control during curve driving. Hum. Factors 28, 211-221.

HaRRIS, J. M. AND BonAS, W. 2002. Optic flow and scene structure do not always contribute to the control of human walking. Vision Res. 42, 1619-1626.

Harris, M. G. and Carré, G. 2001. Is optic flow used to guide walking while wearing a displacing prism? Perception 30, $811-818$.

Kim, N. G. and Turvey, M. T. 1999. Eye-movements and a rule for perceiving direction of heading. Ecol. Psychol. 11, $233-248$.

LAND, M. F. 1998. The visual control of steering. In Vision and Action. L. R. Harris and M. Jenkin, Eds. Cambridge University Press, Cambridge, UK, 163-180.

Lappe, M., Bremmer, F., and Van Den Berg, A. V. 1999. Perception of self-motion from visual flow. Trends Cog. Sci. 3, 329-336.

Loomis, J. M. ANd Beall, A. C. 1998. Visually-controlled locomotion: Its dependence on optic flow, 3-D space perception, and cognition. Ecol. Psychol. 10, 271-285.

Macuga, K. L., Loomis, J. M., Beall, A. C., And Kelly, J. W. In Press. Perception of heading without retinal optic flow. Percept. Psychophys.

MCruer, D. T., Allen, R. W., Weir, D. H., ANd Klein, R. H. 1977. New results in driver steering control models. Hum. Factors 19, 381-397.

Mulder, M., Mulder, J. A., And Stassen, H. G. 2003. A cybernetic analysis of the tunnel-in-the-sky display. In Virtual and Adaptive Environments: Applications, Implications, and Performance Issues, L. J. Hettinger and M. Haas, Eds. Lawrence Erlbaum, Mahwah, NJ, 279-301.

Riemersma, J. B. 1981. Visual control during straight road driving. Acta Psychol. 48, 215-225.

Rogers, B. J. and Dalton, C. 1999. The role of (i) perceived direction and (ii) optic flow in the control of locomotion and for estimating the point of impact. Invest. Ophthalmol. Vis. Sci. 40, S764.

Royden, C. S. 1994. Analysis of misperceived observer motion during simulated eye rotations. Vis. Res. 34, 3215-3222.

Royden, C. S., Banks, M. S., ANd Crowell, J. A. 1992. The perception of heading during eye movements. Nature (London) 360, 583-585.

Royden, C. S., Crowell, J. A., ANd Banks, M. S. 1994. Estimating heading during eye movements. Vis. Res. 34, 3197-3214.

Rushton, S. K., Harris, J. M., Lloyd, M. R., AND Wann, J. P. 1998 . Guidance of locomotion on foot uses perceived target location rather than optic flow. Curr. Biol. 8, 1191-1194.

Stone, L. S. And Perrone, J. A. 1997. Human heading estimation during visually simulated curvilinear motion. Vis. Res. 37, $573-590$.

Turano, K. A., Hicks, J., ANd HaU, L. 2004. Simulated visual field loss in mobile observers: Does retinal location of optic flow matter-Revisited. In Proceedings of the Vision Sciences Society, Sarasota, FL.

Van Den Berg, A. V. 1993. Perception of heading. Nature (London) 365, 497-498.

Wann, J. P. And Land, M. 2000. Steering with or without the flow: is the retrieval of heading necessary? Trends Cog. Sci. 4, 319-324.

Wann, J. P. ANd Land, M. 2001. Heading in the wrong direction: Reply to Fajen and Warren. Trends Cog. Sci. 5, 8-9. 
Wann, J. P. and SwaPp, D. K. 2000. Why you should look where you are going. Nat. Neurosci. 3, 647-648.

Wann, J. P. And Wilkie, R. M. 2004b. How do we control high speed steering? In Optic Flow and Beyond. L. Vaina, S. Beardsley, and S. Rushton, Eds. Kluwer Academic Publishers, Boston, MA, 371-389.

Warren, R. 1976. The perception of egomotion. J. Exp. Psychol. Hum. Percept. Perform. 2, 448-456.

Warren, W. H. 1998. Visually controlled locomotion: 40 years later. Ecol. Psychol. 10, 177-219.

WarRen, W. H. AND Hannon, D. J. 1988. Direction of self-motion is perceived from optical flow. Nature (London) 336, $162-163$.

Warren, W. H., Kay, B. A., Zosh, W. D., Duchon, A. P., And SAhuc, S. 2001. Optic flow is used to control human walking. Nat. Neurosci. 4, 213-216.

Warren, W. H., Mestre, D. R., Blackwell, A. W., and Morris, M. W. 1991. Perception of circular heading from optical flow. J. Exp. Psychol. Hum. Percept. Perform. 17, 28-43.

Warren, W. H., Morris, M. W., and Kalish, M. 1988. Perception of translational heading from optical flow. J. Exp. Psychol. Hum. Percept. Perform. 14, 646-660.

Wilkie, R. M. AND WANN, J. P. 2003a. Eye-movements aid the control of locomotion. J. Vis. 3, 677-684.

Wilkie, R. M. AND WanN, J. P. 2003b. Controlling steering and judging heading: Retinal flow, visual direction, and extraretinal information. J. Exp. Psychol. Hum. Percept. Perform. 29, 363-378.

Wilkie, R. M. AND WANN, J. P. 2006. Judgements of path, not heading, guide locomotion. Journal of Experimental Psychology: Human Perception and Performance 32, 1, 88-96.

Wood, R. M., Harvey, M. A., Young, C. E., Beedie, A., And Wilson, T. 2000. Weighting to go with the flow? Curr. Biol. 10, $545-546$.

Received February 2005; revised November 2005; accepted January 2006 